\title{
MicroRNA-10a suppresses breast cancer progression via PI3K/Akt/mTOR pathway
}

\author{
KONGLIANG KE $^{1^{*}}$ and TINGTING LOU ${ }^{2 *}$ \\ ${ }^{1}$ Department of General Surgery, Ningbo First Hospital, Ningbo, Zhejiang 315010; ${ }^{2}$ Department of Breast Surgery, \\ Ningbo Hangzhou Bay Hospital, Ningbo, Zhejiang 315336, P.R. China
}

Received July 2, 2016; Accepted July 7, 2017

DOI: $10.3892 / \mathrm{ol} .2017 .6930$

\begin{abstract}
Previous studies have demonstrated that microRNA-10a (miR-10a) regulates various opposing biological functions in breast cancer. The aim of the present study was to investigate the exact functions of miR-10a in the pathogenesis of breast cancer. miR-10a expression was initially detected in two human breast cancer cell lines, MCF-7 and MDA-MB-231 and a normal human mammary epithelial cell line MCF-10A. The proliferation, migration and apoptosis of breast cancer cells were analyzed using MTT assays, Transwell assays and flow cytometry, respectively, following transfection of MCF-7 and MDA-MB-231 cells with an miR-10a mimic or anti-miR-10a. The expression of phosphorylated ( $\mathrm{p}-$ )protein kinase B (Akt), p-mammalian target of rapamycin (p-mTOR), p-ribosomal protein S6 kinase $\beta$-1 (p-p70S6K), phosphatidylinositol-4,5-bisphosphate 3-kinase catalytic subunit $\alpha$ (PIK3CA), Cytochrome C (Cyt C), B-cell lymphoma 2 (Bcl-2), BCL-2 associated $\mathrm{X}$, apoptosis regulator (Bax), and cleaved caspase-3 were analyzed by western blotting. The migration of MCF-7 cells pretreated with an mTOR inhibitor CCI-779, was detected using a Transwell assay. Relative miR-10a expression was significantly elevated in MDA-MB-231 breast cancer cells and was at its highest levels in MCF-7 cells. Transfection with the miR-10a mimic significantly inhibited proliferation and migration, and promoted the apoptosis of breast cancer cells. Furthermore, upregulation of miR-10a markedly suppressed the levels of p-Akt, p-mTOR, p-p70S6K, and PIK3CA, and increased the expression of $\mathrm{Cyt} \mathrm{C}$, cleaved caspase-3, and the ratio of Bax/Bcl-2. Anti-miR-10a had the opposite effects. In addition, CCI-779 reversed the effect of anti-miR-10a on the migration of MCF-7 cells in a dose-dependent manner.
\end{abstract}

Correspondence to: Dr Kongliang Ke, Department of General Surgery, Ningbo First Hospital, 59 Liu Ting Street, Ningbo, Zhejiang 315010, P.R. China

E-mail: kekongliang1975@163.com

*Contributed equally

Keywords: microRNA-10a,breast cancer, phosphoinositide-3/protein kinase $\mathrm{B} /$ mammalian target of rapamycin, mitochondrial apoptotic pathway
In conclusion, miR-10a is downregulated in high aggressive breast cancer cells. miR-10a inhibited the proliferation and migration, and promoted apoptosis of breast cancer cells via phosphoinositide/Akt/mTOR signaling, and the mitochondrial apoptotic pathway.

\section{Introduction}

Breast cancer is the most common malignancy in women and a leading cause of cancer-associated mortality globally (1). Furthermore, the high prevalence of women diagnosed at an advanced stage of disease, leads to a poor prognosis (1). Therefore, it is of crucial importance to identify biomarkers associated with predicting prognosis and the progression of breast cancer. MicroRNAs (miRNAs/miRs) are a multifunctional class of small non-coding RNAs that regulate mRNA expression at the post-transcriptional level, by inhibiting protein translation or promoting mRNA degradation (2). An accumulation of evidence suggests that multiple miRNAs are frequently aberrantly expressed in and associated with various processes and phenotypes observed in the initiation and progression of breast cancer.

Previous studies have demonstrated that miR-24-3p was upregulated in breast cancer tissues compared with paired adjacent normal breast tissues (3). Overexpression of miR-24-3p promoted cell proliferation and inhibited apoptosis in human breast cancer cells (3). The overexpression of miR-449a observed in malignant breast tissues, is significantly associated with an increased incidence of patient relapse, a decreased overall survival and disease-free survival (4). Elevated miR-449a expression levels promoted breast cancer cell proliferation, clonogenic survival, migration, and invasion in vitro, indicating that miR-449a promotes the progression of breast cancer (4). miR-30e was reported to be downregulated in plasma and breast cancer tissues, and reduced miR-30e expression was correlated with the clinical stage of breast cancer (5). Another study reported a marked reduction in miR-139-5p in breast cancer cells, the effects of which inhibited cell viability, induced apoptosis, caused cell cycle arrest in the $\mathrm{S}$ phase, and suppressed the migration and invasion of breast cancer cells (6). Furthermore, miR-139-5p mediated drug resistance by regulating the expression of the downstream target gene Notchl (6). miR-503 expression was markedly downregulated in breast cancer tissues and 
cells (7). Overexpression of miR-503 in breast cancer cell lines reduced cell proliferation through inducing G0/G1 cell cycle arrest (7).

Previous studies have demonstrated that miR-10a serves contradictory functions in breast cancer. For example, increased expression of miR-10a in estrogen receptor-positive tumors was associated with a longer relapse-free time following tamoxifen treatment (8). Another previous study revealed that miR-10a was downregulated in breast tumors from patients with early recurrence, which resulted in an overall increased proliferative and angiogenic capacity (9). Khan et al (10) demonstrated that the level of miR-10a expression was significantly decreased in tissues harvested from patients with breast cancer compared with normal and benign tissues. However, a study by Chang et al (11) revealed that elevated miR-10a in relapsed patients was significantly correlated with the hazard ratio of breast cancer recurrence. Furthermore, a previous study revealed that increased expression of miR-10a in MCF-7 human breast adenocarcinoma cells was associated with an inbuilt resistance to cisplatin (12).

The aim of the present study was to initially examine the expression of miR-10a in two human breast cancer cell lines and normal human mammary epithelial cells. Furthermore, the effects of miR-10a on cell proliferation, migration, and apoptosis were assayed. In addition, the mechanisms underlying the biological effects of miR-10a were investigated.

\section{Materials and methods}

Cell culture. The human breast cancer cell lines MDA-MB-231 and MCF-7, and the normal human mammary cell line MCF-10A, were obtained from the American Type Culture Collection (ATCC; Manassas, VA, USA). Cells were cultured in Dulbecco's modified Eagle's medium (DMEM; Invitrogen; Thermo Fisher Scientific, Inc., Waltham, MA, USA), supplemented with $10 \%$ fetal bovine serum (FBS; Hyclone; GE Healthcare Life Sciences, Logan, UT, USA), penicillin $(100 \mathrm{U} / \mathrm{ml})$ and streptomycin $(100 \mu \mathrm{g} / \mathrm{ml})$. Cells were grown at $37^{\circ} \mathrm{C}$ in a humidified chamber at $5 \% \mathrm{CO}_{2}$ in air.

Cell transfection with miR-10a and miR-10a inhibitor. miR-10a mimic, miR-10a inhibitor (anti-miR-10a), miRNA mimic negative control (miR-NC), and miRNA inhibitor negative control (anti-NC) were designed and synthesized by Shanghai GenePharma, Ltd. (Shanghai, China). The sequences were as follows: miR-10a mimic sense, 5'-CAAAUUCGGAUCUAC AGGGUAUU-3' and anti-sense, 5'-UACCCUGUAGAUCCG AAUUUGUG-3'; miR-NC sense, 5'-UUCUCCGAACGU GUCACGUTT-3' and anti-sense, 5'-UACCCUGUAGAUCCG AAUUUGUG-3'; anti-miR-10a, 5'-CACAAAUUCGGAUCU ACAGGGUA-3'; anti-NC, 5'-CAGUACUUUUGUGUAGUA CAA-3'. Cells were cultured to $\sim 60-70 \%$ confluency, following which Lipofectamine ${ }^{\circledR} 2000$ RNAiMAX reagent (Thermo Fisher Scientific, Inc.) was used to transfect the MDA-MB-231 and MCF-7 cells with miR-10a mimic, miR-NC, anti-miR-10a or anti-NC (all $100 \mathrm{nM}$ ) for $48 \mathrm{~h}$ at $37^{\circ} \mathrm{C}$ according to the manufacturer's protocol.

mTOR inhibitor treatment. MCF-7 cells were seeded in 12-well plates at a density of $1 \times 10^{5}$ cells $/ \mathrm{ml}$ and cultured overnight, prior to pretreatment with an mTOR inhibitor CCI-779 $(10 \mu \mathrm{M}$ or $20 \mu \mathrm{M}$, Selleck Chemicals, Houston, TX, USA) for $12 \mathrm{~h}$, followed by transfection with anti-miR-10a or anti-NC as aforementioned.

Cell proliferation assay. Confluent MDA-MB-231 and MCF-7 cells transfected with miR-10a mimic, miR-NC, anti-miR-10a, or anti-NC as aforementioned were seeded in 96-well plates at a density of $2.5 \times 10^{3}$ cells/well. After $48 \mathrm{~h}$ culture, DMEM culture medium was replaced with fresh medium supplemented with $100 \mu \mathrm{l}$ MTT (Sangon Biotech, Co., Ltd., Shanghai, China), followed by incubation for an additional $4 \mathrm{~h}$ at $37^{\circ} \mathrm{C}$. The formazan crystals were dissolved with $150 \mu \mathrm{l}$ DMSO for $10 \mathrm{~min}$. The amount of formazan formed was determined by measuring the absorbance at $550 \mathrm{~nm}$ with a microplate reader (Thermo Fisher Scientific, Inc.).

Cell migration assay. Cell migration activity was detected using a Transwell assay. Briefly, MDA-MB-231 and MCF-7 cells transfected with $100 \mathrm{nM}$ miR-10a mimic, miR-NC, anti-miR-10a or anti-NC were added to the upper chamber of the Transwell assay in $200 \mu \mathrm{l}$ of serum-free DMEM without a Matrigel membrane. DMEM culture medium supplemented with $10 \%$ FBS was added to the lower chamber. The plates were incubated for $48 \mathrm{~h}$ at $37^{\circ} \mathrm{C}$ in $5 \% \mathrm{CO}_{2}$, and non-migrating cells in the upper chambers were removed with a cotton-tipped swab. Cell migration was determined by counting the number of migrated cells under a microscope, following fixation and staining with crystal violet.

Flow cytometry. Apoptosis was determined in MDA-MB-231 and MCF-7 cells using a dual staining method with Annexin V-fluorescein isothiocyanate(FITC) (BDBiosciences, Franklin Lakes, NJ, USA) and 7-amino-actinomycin (7-AAD; BD Biosciences). Briefly, $1 \times 10^{6}$ cells were harvested at 48 h by trypsin/EDTA digestion following transfection with miR-10a mimic or miR-NC mimic. Annexin V-FITC and 7-AAD were added to the cell suspension according to the manufacturer's protocol. Cells were analyzed using a FACSCalibur flow cytometer (Becton Dickinson; BD Biosciences) using Cell-Quest Pro software (version 5.1; BD Biosciences, Franklin Lakes, NJ, USA). Apoptosis was assessed by counting the number of early and late apoptotic cells and determined by relative apoptotic changes.

Reverse transcription quantitative polymerase chain reaction (RT-qPCR). Total RNA was extracted from cells using the RNAisoPlus reagent (Takara, Bio, Inc., Otsu, Japan) according to the manufacturer's protocol. A total of $1 \mu \mathrm{g}$ mRNA was reverse transcribed to cDNA with oligo (dT) primers using TaqMan microRNA Reverse Transcription kit (Applied Biosystems; Thermo Fisher Scientific, Inc., Waltham, MA, USA) according to the protocol of the manufacturer. qPCR analysis was performed using SYBR-Green (Takara Biotechnology, Co., Ltd, Dalian, China) and the ABI PRISM 7500 Sequence Detection System (Applied Biosystems; Thermo Fisher Scientific, Inc.). The primer sequences for miR-10a and Phosphatidylinositol-4,5-Bisphosphate 3-Kinase Catalytic Subunit $\alpha$ (PIK3CA) were as follows: miR-10a forward 5'-GGATACCCTGTAGATCCGAA-3' and reverse 


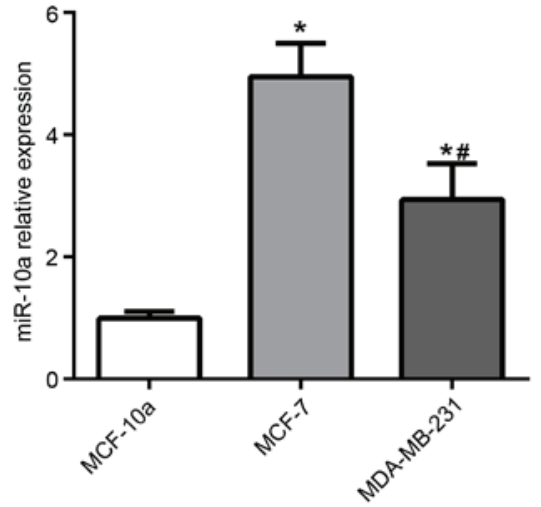

Figure 1. Expression of miR-10a in MCF-10A, MCF-7, and MDA-MB-231 cells was detected by reverse transcription-quantitative polymerase chain reaction. Each experiment is representative of three independent repeats. Each value represents the mean \pm standard deviation. ${ }^{*} \mathrm{P}<0.05$ vs. MCF-10A; ${ }^{\#} \mathrm{P}<0.05$ vs. MCF-7. miR-10a, microRNA-10a.

5'-CAGTGCGTGTCGTGGAGT-3'; PIK3CA forward, 5'-GCA TACATTCGAAAGACC-3' and reverse, 5'-CTCAGTTAT CTTTTCAG-3'. The expression level of PIK3CA was calculated using the $2^{-\Delta \Delta \mathrm{Cq}}$ method with normalization to GAPDH (forward, 5'-CTTCACCACCATGGAGAAGGC-3' and reverse, 5'-GGCATGGACTGTGGTCATGAG-3') (13).

Western blot analysis. Cells were harvested for total protein extraction and lysed with $100 \mu 1$ LRIPA lysis buffer for $30 \mathrm{~min}$ on ice. Total protein concentrations were analyzed using an Easy Protein Quantitative kit (BCA; Transgen Biotech Co, Ltd, Beijing, China). Total proteins (30 $\mu \mathrm{g} / \mathrm{lane})$ were separated using SDS-PAGE on a $10 \%$ gel and transferred to nitrocellulose membranes. The membranes were blocked using $5 \%$ non-fat milk for $2 \mathrm{~h}$ at room temperature and incubated with the following antibodies overnight at $4^{\circ} \mathrm{C}$ : Anti-PIK3CA (1:5,000; cat. no. ab40776; Abcam, Cambridge, MA, USA) anti-p-Akt (1:5,000; cat. no. ab81283; Abcam), anti-p-mTOR (1:1,000; cat. no. ab1093; Abcam), anti-p-ribosomal protein S6 kinase $\beta$-1 (p-p70S6K; 1:500; cat. no. ab5231; Abcam), anti-B-cell lymphoma 2 (Bcl-2; 1:1,000; cat. no. 15071; Cell Signaling Technology, Inc., Danvers, MA, USA), anti-BCL-2 associated-X, apoptosis regulator (Bax; 1:1,000; cat. no. 2772; Cell Signaling Technology, Inc.), anti-cleaved caspase-3 (1:1,000; cat. no. 9661; Cell Signaling Technology, Inc.), and anti-cytochrome-C (Cyt-C; 1:1,000; cat. no. 4280; Cell Signaling Technology, Inc.). Following washing with TBST, the membranes were incubated with goat anti-rabbit IgG horseradish peroxidase-conjugated secondary antibodies (1:5,000; cat. no. sc-2004; Santa Cruz Biotechnology, Santa Cruz, CA, USA) for $1 \mathrm{~h}$ at room temperature and proteins were visualized using an enhanced chemiluminescence reagent (EMD Millipore, Billerica, MA, USA). Data were quantified by using Image J software (version $1.48 \mathrm{u}$; National Institutes of Health, Bethesda, MD, USA).

Statistical analysis. Data were expressed as the mean \pm standard deviation. The statistical analyses were performed using one-way analysis of variance followed by Least Significant Difference test or unpaired Student's t-test with SPSS software (version 13.0; SPSS, Inc., Chicago, IL, USA). $\mathrm{P}<0.05$ was considered to indicate a statistically significant difference.

\section{Results}

miR-10a is downregulated in high aggressive breast cancer cell line. To determine the function of miR-10a in breast cancer, miR-10a expression was examined in MCF-10A human mammary cells, MCF-7, and MDA-MB-231 breast cancer cell lines. The expression of miR-10a was significantly increased in MCF-7 and MDA-MB-231 cells compared with MCF-10A cells, as presented in Fig. 1. Furthermore, the level of miR-10a expression was significantly lower in MDA-MB-231 cells compared with MCF-7 cells.

miR-10a inhibits breast cancer cell proliferation and migration, and induces cell apoptosis. The results from the present study revealed that miR-10a was significantly downregulated in MDA-MB-231 cells compared with MCF-7 cells (Fig. 1), which may indicate that miR-10a functions as a tumor suppressor. Therefore, the effects of miR-10a on the proliferation, migration and apoptosis of breast cancer cells via the upregulation/downregulation of miR-10a were determined. The results presented in Fig. 2A revealed that a slower rate of cell proliferation was observed in cells transfected with an miR-10a mimic compared with miR-NC mimic in breast cancer cell lines. However, transfection of anti-miR-10a resulted in the opposite effect. Furthermore, the miR-10a mimic significantly dampened the migration of MCF-7 and MDA-MB-231 cells, and anti-miR-10a enhanced cell migration compared with the control (Fig. 2B). In addition, transfection with the miR-10a mimic significantly enhanced the apoptosis of the breast cancer cell lines compared with the control (Fig. 2C). The data presented indicated that miR-10a is efficient at suppressing the proliferation and migration and facilitating the apoptosis of breast cancer cells.

miR -10 a regulates the PI3K/Akt/mTOR pathway. Previous studies have demonstrated that the PI3K/Akt/mTOR pathway is an important target in breast cancer (14). To evaluate whether the inhibitory effects of miR-10a on the proliferation and migration of breast cancer cells and the enhanced apoptotic effects are regulated via the PI3K/Akt/mTOR pathway, the expression of p-Akt, p-mTOR, and p-p70S6K was determined in breast cancer cells. Transfection with the miR-10a mimic significantly suppressed the levels of p-Akt, p-mTOR, and p-p70S6K in MDA-MB-231 cells (Fig. 3A and $\mathrm{B})$. In addition, anti-miR-10a markedly increased the expression of p-Akt, p-mTOR, and p-p70S6K in MCF-7 cells (Fig. 3A and C). Additionally, the effects of pretreatment with an mTOR inhibitor CCI-779 $(0,10$ or $20 \mu \mathrm{M})$ for $24 \mathrm{~h}$ prior to transfection with anti-miR-10a in MCF-7 cells, decreased the effect of anti-miR-10a on the migration of MCF-7 cells in a dose-dependent manner (Fig. 3D). These data indicated that miR-10a functions via the PI3K/Akt/mTOR signaling pathway in breast cancer cells.

miR-10a decreases PIK3CA expression in MDA-MB-231 cells. It has previously been demonstrated that PIK3CA, the central component of the PI3K pathway, is a direct target gene of miR-10a (15). Therefore, PIK3CA expression was 

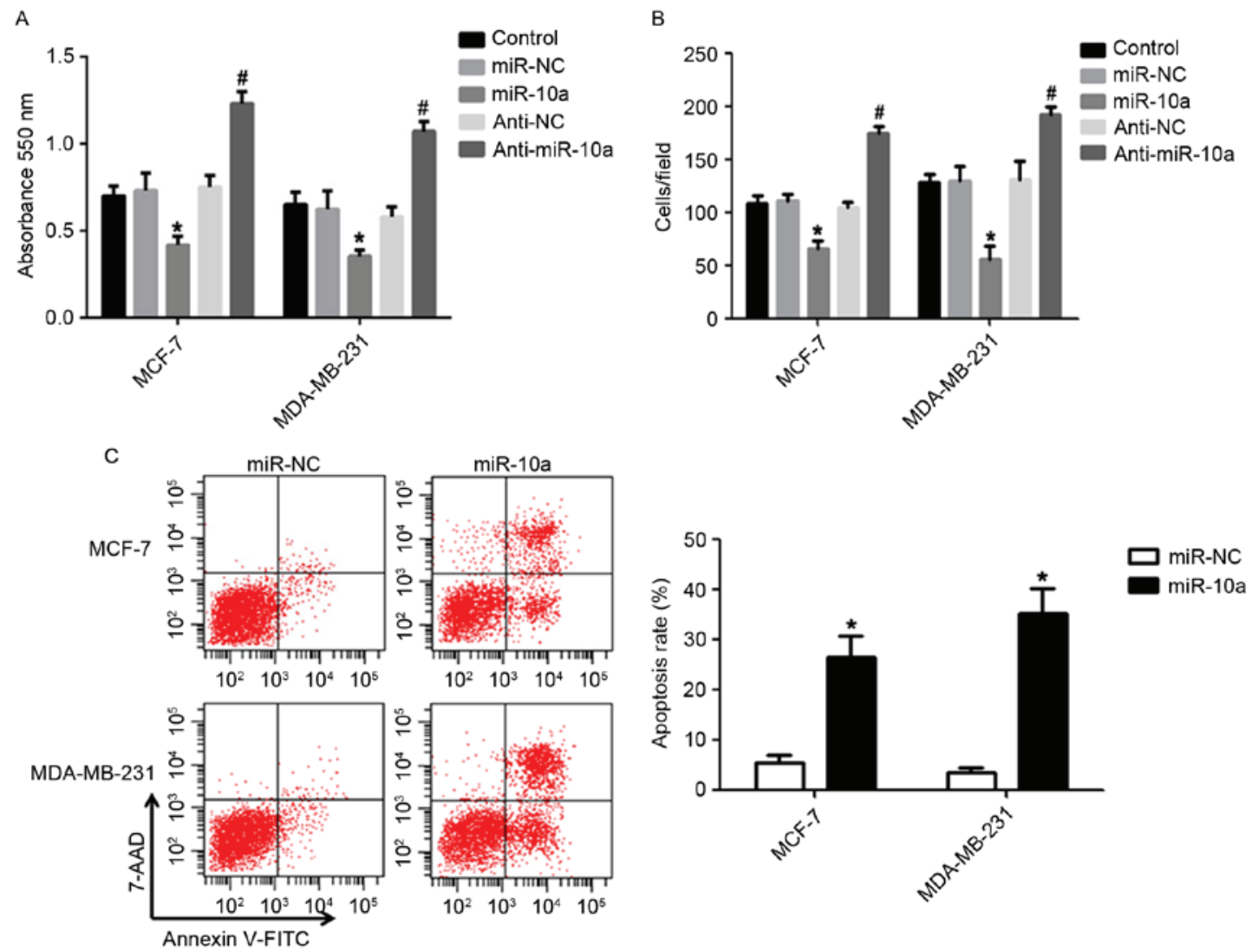

Figure 2. Effects of miR-10a on the proliferation, migration, and apoptosis of breast cancer cells. (A) The proliferation of MCF-7 and MDA-MB-231 cells transfected with miR-10a or anti-miR-10a was detected by MTT analysis. (B) Transwell analysis for the migration of MCF-7 and MDA-MB-231 cells. (C) The apoptosis of MCF-7 and MDA-MB-231 cells transfected with miR-10a was analyzed by flow cytometry. Each experiment was independently repeated three times. Values represent the mean \pm standard deviation, ${ }^{*} \mathrm{P}<0.05$ vs. miR-NC, ${ }^{\#} \mathrm{P}<0.05$ vs. anti-NC. miR, microRNA; NC, negative control.

A

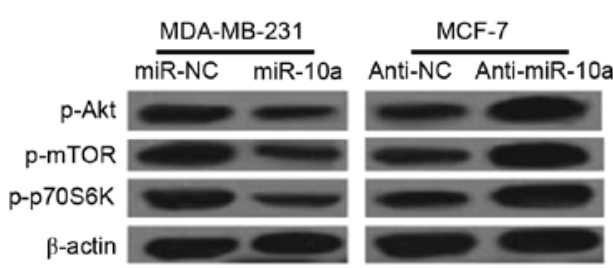

C

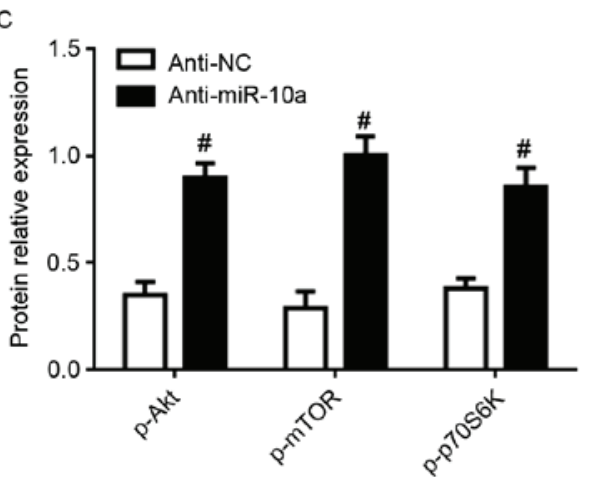

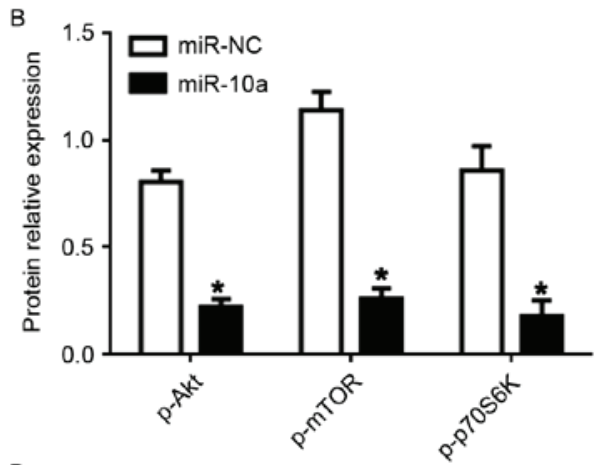

D

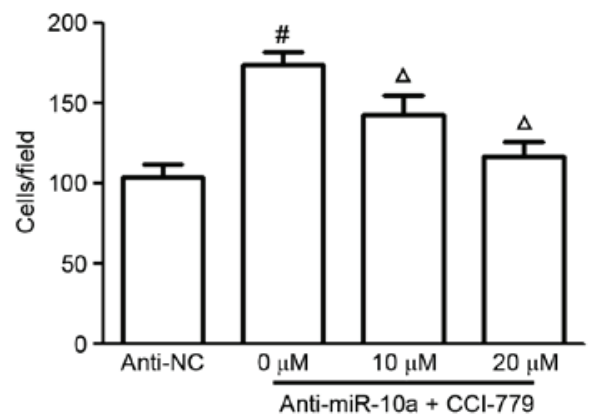

Figure 3. Effects of miR-10a on PI3K/Akt/mTOR signaling. (A) Western blot analysis for the protein expression of p-Akt, p-mTOR, p-p70S6K in MDA-MB-231 and MCF-7 cells transfected with miR-10a or anti-miR-10a. Statistical analysis of p-AKT, mTOR and p-P-70S6K expression in cells transfected with (B) miR-10a mimic and miR-NC and (C) anti-miR-10a and anti-NC. (D) Cell migration of MCF-7 cells pretreated with an mTOR inhibitor CCI-779 (0, 10 or $20 \mu \mathrm{M}$ ) for $24 \mathrm{~h}$ followed by transfection with anti-miR-10a, determined by Transwell analysis. Each experiment is representative of three independent repeats. Each value represents the mean \pm standard deviation. ${ }^{*} \mathrm{P}<0.05$ vs. miR-NC, ${ }^{*} \mathrm{P}<0.05$ vs. anti-NC, ${ }^{\Delta} \mathrm{P}<0.05$ vs. anti-miR-10a. miR-10a, microRNA-10a; P13K, phosphatidylinositol-3-kinase; Akt, protein kinase B; mTOR, mammalian target of rapamycin; p70S6K, ribosomal protein S6 kinase $\beta-1$; p-, phosphorylated. 
A

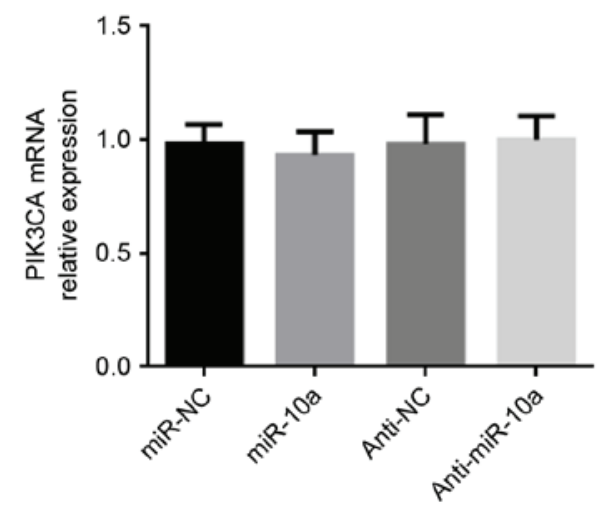

B
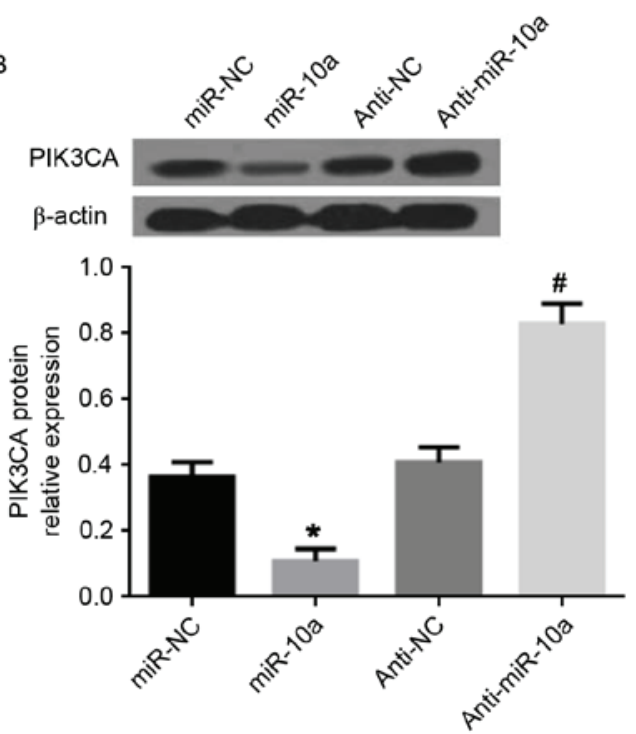

Figure 4. Effects of miR-10a on PIK3CA expression in MDA-MB-231 cells. (A) mRNA expression of PIK3CA in MDA-MB-231 cells transfected with miR-10a mimic or miR-NC mimic, analyzed by RT-qPCR. (B) Western blot analysis of PIK3CA protein expression. Each experiment was independently repeated three times. Each value represents the mean \pm standard deviation, ${ }^{~} \mathrm{P}<0.05$ vs. miR-NC, ${ }^{~} \mathrm{P}<0.05$ vs. anti-NC. miR, microRNA; PIK3CA, phosphatidylinositol-4,5-bisphosphate 3-kinase catalytic subunit $\alpha$; RT-qPCR, reverse transcription quantitative polymerase chain reaction; NC, negative control.

A

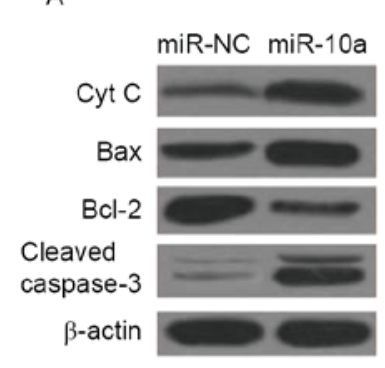

B

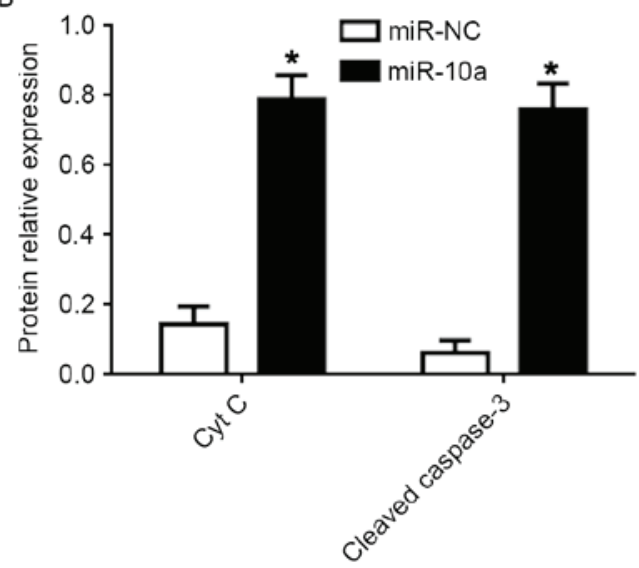

C

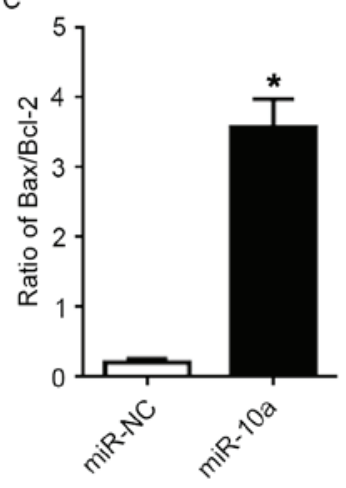

Figure 5. Effects of miR-10a on the mitochondrial apoptotic pathway in breast cancer cells. (A) Western blot analysis of Cyt C, Bax, Bcl-2, and cleaved caspase-3 protein expression in MCF-7 cells. Quantification of the protein levels of (B) Cyt C and cleaved caspase-3, and (C) the ratio of Bax/Bcl-2. Each value represents the mean \pm standard deviation, ${ }^{*} \mathrm{P}<0.05$ vs. miR-NC. miR, microRNA; Bax, BCL-2 associated X, apoptosis regulator; Bcl-2, B-cell lymphoma 2; Cyt C, cytochrome C; NC, negative control.

investigated following transfection of the miR-10a mimic or anti-miR-10a in MDA-MB-231 cells. The data presented in Fig. 4 demonstrated that the miR-10a mimic did not affect PIK3CA gene expression, but significantly reduced PIK3CA at the protein level. Conversely, anti-miR-10a increased PIK3CA at the protein level, whereas no effect was observed on PIK3CA gene expression. Thus, it is suggested that miR-10a inhibits PIK3CA expression at the posttranscriptional level to suppress PI3K/Akt/mTOR signaling in breast cancer cells.

miR-10a induces the mitochondrial apoptotic pathway. The $\mathrm{PI} 3 \mathrm{~K} / \mathrm{Akt} / \mathrm{mTOR}$ pathway is associated with the mitochondrial mediated apoptosis in tumor cells including human breast cancer cells $(16,17)$. To further investigate the intrinsic pathway of apoptosis, the protein expression of $\mathrm{Cyt} \mathrm{C}, \mathrm{Bcl}-2$,
Bax, and cleaved caspase- 3 was determined in MCF-7 cells following transfection of miR-10a mimic. Transfection with the miR-10a mimic significantly increased the expression of Cyt $\mathrm{C}$ and cleaved caspase-3 (Fig. 5A and B). Furthermore, the ratio of $\mathrm{Bax} / \mathrm{Bcl}-2$ was significantly increased following overexpression of the miR-10a, compared with miR-NC mimic (Fig. 5C).

\section{Discussion}

The present study aimed to investigate the involvement of miR-10a in the pathogenesis of breast cancer. Previous studies have demonstrated that the expression of miR-10a is contradictory in breast cancer $(10,11)$. In the present study, the expression of miR-10a was initially detected in MCF-7 and MDA-MB-231 breast cancer cells and MCF-10A normal mammary cells. The 
data presented demonstrated that the expression of miR-10a was increased in breast cancer cell lines. Notably, the level of miR-10a expression was lower in the more aggressive breast cancer cell line MDA-MB-231 compared with the less aggressive MCF-7 cells (Fig. 1). The loss of miR-10a expression in gastric cancer highlighted a potential tumor suppressor function for miR-10a (18). Thus, we speculated that miR-10a may function as a tumor suppressor in breast cancer, and miR-10a expression levels may be considered as a predictor for the progression of breast cancer.

To evaluate the exact function of miR-10a in breast cancer, the induced upregulation and downregulation of miR-10a expression were studies in breast cancer cells. Transfection of miR-10a markedly inhibited proliferation and migration, whilst promoting the apoptosis of breast cancer cells. Conversely, transfection with anti-miR-10a induced the opposite effects on cell proliferation and migration (Fig. 2). The $\mathrm{PI} 3 \mathrm{~K} / \mathrm{Akt} / \mathrm{mTOR}$ signaling pathway serves a key regulatory function in cell survival, proliferation, migration, metabolism, and apoptosis, and its aberrant activation has been observed in multiple types of cancer, including breast cancer (16). p70S6K is the downstream effector of mTOR. The results of the present study demonstrated that p-Akt, p-mTOR, and p-p70S6K expression was significantly decreased following overexpression of miR-10a in MDA-MB-231 cells. The role of miR-10a on PI3K/Akt/mTOR signalling subsequent to downregulation of miR-10a in MCF-7 cells was also confirmed, and the results indicated that the levels of p-Akt, p-mTOR, and p-p70S6K were enhanced by anti-miR-10a expression in MCF-7 cells. In addition, an mTOR inhibitor, CCI-779, reversed the effect of anti-miR-10a on the migration of $\mathrm{MCF}-7$ cells in a dose-dependent manner (Fig. 3). The results from the present study indicated that miR-10a suppresses proliferation and migration in, yet promotes the apoptosis of breast cancer cells via impeding PI3K/Akt/mTOR signaling.

PIK3CA is a fundamental molecule of the PI3K/Akt/mTOR pathway. Enhanced activation of PIK3CA has been observed in a high proportion of several types of human tumors, and is associated with mammary tumorigenesis and angiogenesis (19). In addition, PIK3CA is relevant in terms of resistance to endocrine or anti-HER2 therapies $(20,21)$. Previous studies have demonstrated that miR-10a directly suppresses the expression of PIK3CA by targeting the 3y-untranslated region of the gene in airway smooth muscle cells to control cell proliferation (15). To confirm whether miR-10a functions through targeting PIK3CA, the gene and protein expression of PIK3CA following transfection with miR-10a mimic or anti-miR-10a in MDA-MB-231 cells was determined. The results of the present study revealed that miR-10a markedly decreased the protein levels of PIK3CA, however no detectable changes were observed at the mRNA level, compared with the control mimic. Notably, the protein expression of PIK3CA was upregulated in response to anti-miR-10a, but no changes were observed at the mRNA level (Fig. 4). The results from the present study indicated that miR-10a targets PIK3CA expression to inhibit the PI3K/Akt/mTOR pathway in breast cancer cells.

To further investigate the mechanism of miR-10a on the apoptosis of breast cancer cells, the activity of the mitochondrial apoptotic pathway in MCF-7 cells. Mitochondria are known to play a central role in cell apoptosis. The mitochondrial mediated apoptotic pathway is upstream of caspase activation and is regulated by the Bcl-2 family of proteins. Any imbalance in the expression of Bcl-2 and Bax protein will disrupt the outer membrane of mitochondria (22). Previous studies have demonstrated that the increase of Bax/Bcl-2 modulates mitochondrial permeability, releasing Cyt $\mathrm{C}$ from mitochondria (23). In the present study, transfection with the miR-10a mimic significantly increased the expression of Cyt $\mathrm{C}$ and cleaved caspase-3, and enhanced the $\mathrm{Bax} / \mathrm{Bcl}-2$ ratio. Taken together, the results of the present study indicated that miR-10 regulates breast cancer cell apoptosis via the mitochondrial apoptotic pathway.

In conclusion, to the best of our knowledge, the present study is the first to report that miR-10a is downregulated in an aggressive breast cancer cell line, which indicates that miR-10a expression may be a potential predictor of the development of breast cancer. Furthermore, miR-10a suppresses proliferation and migration in, and promotes apoptosis of, breast cancer cells through targeting the PI3K/Akt/mTOR pathway and promoting the mitochondrial mediated apoptotic pathway; which indicates that miR-10a functions as a suppressor in the progression of breast cancer.

\section{References}

1. Lauby-Secretan B, Scoccianti C, Loomis D, Benbrahim-Tallaa L, Bouvard V, Bianchini F and Straif K; International Agency for Research on Cancer Handbook Working Group: Breast-cancer screening-viewpoint of the IARC Working Group. N Engl J Med 372: 2353-2358, 2015.

2. Bartel DP: MicroRNAs: Genomics, biogenesis, mechanism and function. Cell 116: 281-297, 2004.

3. Lu K, Wang J, Song Y, Zhao S, Liu H, Tang D, Pan B, Zhao H and Zhang Q: miRNA-24-3p promotes cell proliferation and inhibits apoptosis in human breast cancer by targeting p27Kip1. Oncol Rep 34: 995-1002, 2015.

4. Shi W, Bruce J, Lee M, Yue S, Rowe M, Pintilie M, Kogo R, Bissey PA, Fyles A, Yip KW and Liu FF: MiR-449a promotes breast cancer progression by targeting CRIP2. Oncotarget 7: 18906-18918, 2016.

5. Lin Z, Li JW, Wang Y, Chen T, Ren N, Yang L, Xu W, He H, Jiang Y, Chen X, et al: Abnormal miRNA-30e Expression is Associated with Breast Cancer Progression. Clin Lab 62: 121-128, 2016.

6. Zhang HD, Sun DW, Mao L, Zhang J, Jiang LH, Li J, Wu Y, Ji H, Chen W, Wang J, et al: MiR-139-5p inhibits the biological function of breast cancer cells by targeting Notch1 and mediates chemosensitivity to docetaxel. Biochem Biophys Res Commun 465: 702-713, 2015.

7. Long J, Ou C, Xia H, Zhu Y and Liu D: MiR-503 inhibited cell proliferation of human breast cancer cells by suppressing CCND1 expression. Tumour Biol 36: 8697-8702, 2015.

8. Hoppe R, Achinger-Kawecka J, Winter S, Fritz P, Lo WY, Schroth W and Brauch H: Increased expression of miR-126 and miR-10a predict prolonged relapse-free time of primary oestrogen receptor-positive breast cancer following tamoxifen treatment. Eur J Cancer 49: 3598-3608, 2013.

9. Pérez-Rivas LG, Jerez JM, Carmona R, de Luque V, Vicioso L, Claros MG, Viguera E, Pajares B, Sánchez A, Ribelles N, et al: A microRNA signature associated with early recurrence in breast cancer. PLoS One 9: e91884, 2014.

10. Khan S, Wall D, Curran C, Newell J, Kerin MJ and Dwyer RM: MicroRNA-10a is reduced in breast cancer and regulated in part through retinoic acid. BMC Cancer 15: 345, 2015.

11. Chang CH, Fan TC, Yu JC, Liao GS, Lin YC, Shih AC, Li WH and Yu AL: The prognostic significance of RUNX2 and miR-10a/10b and their inter-relationship in breast cancer. J Transl Med 12: 257, 2014.

12. Pogribny IP, Filkowski JN, Tryndyak VP, Golubov A, Shpyleva SI and Kovalchuk O: Alterations of microRNAs and their targets are associated with acquired resistance of MCF-7 breast cancer cells to cisplatin. Int J Cancer 127: 1785-1794, 2010. 
13. Livak KJ and Schmittgen TD: Analysis of relative gene expression data using real-time quantitative PCR and the 2(-Delta Delta C(T)) Method. Methods 25: 402-408, 2001

14. Guo Y, Chang H, Li J, Xu XY, Shen L, Yu ZB and Liu WC: Thymosin alpha 1 suppresses proliferation and induces apoptosis in breast cancer cells through PTEN-mediated inhibition of $\mathrm{PI3K} / \mathrm{Akt} / \mathrm{mTOR}$ signaling pathway. Apoptosis 20: 1109-1121, 2015.

15. Hu R, Pan W, Fedulov AV, Jester W, Jones MR, Weiss ST, Panettieri RA Jr, Tantisira K and Lu Q: MicroRNA-10a controls airway smooth muscle cell proliferation via direct targeting of the PI3Kinase pathway. FASEB J 28: 2347-2357, 2014.

16. Kamal A, Lakshma Nayak V, Nagesh N, Vishnuvardhan MV and Subba Reddy NV: Benzo [b]furan derivatives induces apoptosis by targeting the PI3K/Akt/mTOR signaling pathway in human breast cancer cells. Bioorg Chem 66: 124-131, 2016.

17. Wani ZA, Guru SK, Rao AV, Sharma S, Mahajan G, Behl A, Kumar A, Sharma PR, Kamal A, Bhushan S and Mondhe DM: A novel quinazolinone chalcone derivative induces mitochondrial dependent apoptosis and inhibits PI3K/Akt/mTOR signaling pathway in human colon cancer HCT-116 cells. Food Chem Toxicol 87: 1-11, 2016

18. Jia H, Zhang Z, Zou D, Wang B, Yan Y, Luo M, Dong L, Yin H, Gong B, Li Z et al: MicroRNA-10a is down-regulated by DNA methylation and functions as a tumor suppressor in gastric cancer cells. PLoS One 9: e88057, 2014.
19. Renner O, Blanco-Aparicio C, Grassow M, Canamero M, Leal JF and Carnero A: Activation of phosphatidylinositol 3-kinase by membrane localization of p110alpha predisposes mammary glands to neoplastic transformation. Cancer Res 68: 9643-9653, 2008.

20. Egeland NG, Lunde S, Jonsdottir K, Lende TH, Cronin-Fenton D, Gilje B, Janssen EA and Søiland H: The role of MicroRNAs as predictors of response to tamoxifen treatment in breast cancer patients. Int J Mol Sci 16: 24243-24275, 2015.

21. Yang SX, Polley E and Lipkowitz S: New insights on PI3K/AKT pathway alterations and clinical outcomes in breast cancer. Cancer Treat Rev 45: 87-96, 2016.

22. Suen DF, Norris KL and Youle RJ: Mitochondrial dynamics and apoptosis. Genes Dev 22: 1577-1590, 2008.

23. Breckenridge DG and Xue D: Regulation of mitochondrial membrane permeabilization by BCL-2 family proteins and caspases. Curr Opin Cell Biol 16: 647-652, 2004. 